\title{
Predictors of Poor Outcome after Successful Mechanical Thrombectomy in Patients with Acute Anterior Circulation Stroke
}

\author{
Yosuke Tajima ${ }^{1}$ Michihiro Hayasaka ${ }^{1}$ Koichi Ebihara ${ }^{1}$ Masaaki Kubota ${ }^{1}$ Sumio Suda ${ }^{1}$ \\ ${ }^{1}$ Department of Neurosurgery, Kimitsu Chuo Hospital, \\ Kisarazu, Japan \\ Address for correspondence Yosuke Tajima, MD, PhD, Department \\ of Neurosurgery, Kimitsu Chuo Hospital, 1010 Sakurai, Kisarazu \\ 4-9-1 292-8535, Japan (e-mail: tajima5615@yahoo.co.jp). \\ J Clin Interv Radiol ISVIR 2017;1:139-143.
}

\begin{abstract}
Successful revascularization is one of the main predictors of a favorable clinical outcome after mechanical thrombectomy. However, even if mechanical thrombectomy is successful, some patients have a poor clinical outcome. This study aimed to investigate the clinical, imaging, and procedural factors that are predictive of poor clinical outcomes despite successful revascularization after mechanical thrombectomy in patients with acute anterior circulation stroke. The authors evaluated 69 consecutive patients (mean age, 74.6 years, 29 women) who presented with acute ischemic stroke due to internal cerebral artery or middle cerebral artery occlusions and who were successfully treated with mechanical thrombectomy between July 2014 and November 2016. A good outcome was defined as a modified Rankin Scale score of 0 to 2 at 3 months after treatment. The associations between the clinical, imaging, and procedural factors and poor outcome were evaluated using logistic regression analyses. Using multivariate analyses, the authors found that the preoperative National Institute of Health Stroke Scale (NIHSS) score (odds ratio [OR], 1.152; 95\% confidence interval $[\mathrm{Cl}], 1.004-1.325 ; p=0.028)$, the diffusion-weighted imaging Alberta Stroke Program Early Computed Tomography Score (DWI-ASPECTS) (OR, 0.604;

\section{Keywords}

- acute ischemic stroke

- mechanical thrombectomy

- poor outcome 95\% Cl, 0.412-0.882; $p=0.003$ ), and a Thrombolysis in Cerebral Infarction ( $\mathrm{TICl}) 2 \mathrm{~b}$ classification (OR, $4.521 ; 95 \% \mathrm{Cl}, 1.140-17.885 ; p=0.026$ ) were independent predictors of poor outcome. Complete revascularization to reduce the infarct volume should be performed, especially in patients with a high DWI-ASPECTS, to increase the likelihood of a good outcome.
\end{abstract}

\section{Introduction}

In the modern stent retriever era, the results of mechanical thrombectomy for acute cerebral infarction have improved. Indeed, a series of randomized controlled trials demonstrating the effectiveness of mechanical thrombectomy for acute anterior circulation stroke have been reported. ${ }^{1-5}$ Studies examining the predictors of poor outcome or mortality concluded that successful revascularization is a potent predictor of a favorable

received

September 25, 2017

accepted after revision

October 30, 2017

published online

December 14, 2017 10.1055/s-0037-1612600. ISSN 2457-0214. clinical outcome. ${ }^{6-8}$ However, some patients have a poor clinical outcome, including mortality, despite successful mechanical thrombectomy. Currently, the factors related to the poor outcomes remain unknown.

Therefore, the present study aimed to investigate the clinical, imaging, and procedural factors that are predictive of poor clinical outcomes despite successful revascularization after mechanical thrombectomy in patients with acute anterior circulation stroke.
Copyright $\odot 2017$ by Indian Society of Vascular and Interventional Radiology
License terms

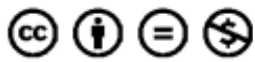




\section{Patients and Methods}

\section{Patients}

In this study, we evaluated the data from 69 consecutive patients who presented with acute ischemic stroke due to internal cerebral artery (ICA) or middle cerebral artery (MCA) occlusions and who were successfully treated with mechanical thrombectomy at our hospital between July 2014 and November 2016.

\section{Assessments}

The following comorbidities were registered: atrial fibrillation, hypertension, diabetes, dyslipidemia, and active smoking. Stroke severity at presentation was described using the National Institutes of Health Stroke Scale (NIHSS) on admission. Infarct size was estimated with the diffusion-weighted imaging Alberta Stroke Program Early Computed Tomography Score (DWI-ASPECTS). The diagnosis of ICA or MCA occlusion had to be proven on magnetic resonance angiography (MRA) or digital subtraction angiography images in all patients. Furthermore, we collected the following clinical, imaging, and procedural data: use of intravenous thrombolysis, time from onset to admission, time from puncture to revascularization, time from onset to revascularization, revascularization status, and intracranial hemorrhage (ICH) on posttreatment computed tomographic (CT) scans. Revascularization status was assessed on the final angiogram and was classified according to the Thrombolysis in Cerebral Infarction (TICI) scale; successful revascularization was defined as a modified TICI grade of $2 b$ or 3. As mentioned previously, we assessed ICHs on posttreatment CT images. A symptomatic ICH was defined as any ICH that caused neurologic deterioration (increase of $\geq 4$ points in the NIHSS or decrease of 1 point in the level of consciousness element of the NIHSS). The clinical outcome was assessed using the modified Rankin Scale (mRS) at 3 months after treatment. A good clinical outcome was defined as an mRS score of 0 to 2 .

\section{Endovascular Therapy}

Endovascular therapy was performed under conscious sedation using a femoral artery approach. A 9F Optimo (Tokai Medical Product) was inserted into the target carotid artery. The techniques used for the subsequent mechanical thrombectomy were determined by each neurointerventionist (Y.T., M.H., and K.E.). Two techniques were used, including the direct aspiration first-pass thrombectomy (ADAPT) technique with a 5MAX ACE reperfusion catheter (Penumbra) and the stent-based thrombectomy technique.

In cases in which the ADAPT technique was used, a coaxial system consisting of a 5MAX ACE reperfusion catheter (Penumbra), Marksman microcatheter (Covidien), and 0.016-in microguidewire (GT; Terumo) was inserted in the 9F Optimo (Tokai Medical Product). Once the reperfusion catheter was immediately adjacent to the clot, the microcatheter and microguidewire were removed. Thereafter, the aspiration tubing was connected directly to the hub of the reperfusion catheter, and the Penumbra aspiration pump was turned on. After a waiting period of 90 seconds, the reperfusion catheter was slowly withdrawn under continuous aspiration.
In cases in which stent-based thrombectomy was used, the Marksman microcatheter (Covidien) was carefully navigated through the thromboembolic occlusion over the 0.016-in microguidewire (Terumo). A microcatheter angiographic run was subsequently performed to confirm the correct position of the microcatheter distal to the thrombus. The microwire was replaced by the Trevo Pro clot retriever (ProVue or VP; Stryker). Once the Trevo Pro clot retriever was fully deployed, angiographic runs were performed to check for correct placement and expansion of the device and flow restoration. The fully deployed Trevo Pro clot retriever was held in place for 5 minutes, and then the stent and microcatheter were removed. During retrieval, manual aspiration was performed with a $50-\mathrm{mL}$ syringe to reverse the flow and to remove any clot debris in the guide catheter lumen. If thrombus removal was unsuccessful, both the Penumbra catheter and the Trevo Pro clot retriever were used.

\section{Statistical Analysis}

Statistical evaluations were performed using JMP 13.1.0 (SAS Institute Inc.). For the univariate analyses, data were compared using Student's $t$-tests for continuous variables or chi-square tests for categorical variables. To determine the predictors of a poor clinical outcome, a forward, stepwise logistic regression analysis that included all variables with a $p$ value of $<0.2$ in the univariate analyses was performed. Statistical significance was set at $p<0.05$.

\section{Results}

\section{Baseline Characteristics of the Patients}

In the defined period (July 2014 to November 2016), 40 men and 29 women (mean age, 74.6 years; range, 55-93 years) met the criteria for the present study and were retrospectively included. The mean NIHSS score at presentation was 20.3 (range, 8-31). Of the 69 included patients, 35 (50.7\%) had atrial fibrillation, 36 (52.2\%) had hypertension, and 15 (21.7\%) had diabetes mellitus. The patients' mean DWI-ASPECTS was 7.5 (range, 3-11). Among the 69 patients, the occluded vessel was the ICA in 24 patients (34.8\%), the MCA M1 portion in 41 patients (59.4\%), and the MCA M2 portion in 4 patients (5.8\%). The detailed baseline and clinical characteristics of the patients are shown in -Table 1.

\section{Clinical Outcomes}

At 3 months after treatment, 46 patients (66.7\%) were functionally independent (mRS score of $0-2$ ), 3 (4.3\%) had moderate disability (mRS score of 3 ), 16 (23.2\%) were severely disabled (mRS score of 4 ), and 4 (5.8\%) were deceased (-Fig. 1). The 46 patients who were functionally independent according to the mRS score were placed in the good outcome group, whereas the remaining 23 patients were placed in the poor outcome group.

\section{Procedural Results}

Detailed procedural results are shown in - Table 2. In 23 of the 69 patients (33.3\%), tissue plasminogen activator was intravenously injected. Of the 69 patients, 50 (72.5\%) received treatment only via the ADAPT technique, 25 (36.2\%) 
Table 1 Baseline characteristics of patients who underwent mechanical thrombectomy for acute ischemic stroke

\begin{tabular}{|c|c|c|c|c|}
\hline & $\begin{array}{l}\text { All } \\
(n=69)\end{array}$ & $\begin{array}{l}\text { Good outcome } \\
(n=46)\end{array}$ & $\begin{array}{l}\text { Poor outcome } \\
(n=23)\end{array}$ & $p$ Value \\
\hline \multicolumn{5}{|l|}{ Demographics } \\
\hline Age, years, mean $\pm S D$ & $74.6 \pm 9.2$ & $72.6 \pm 9.3$ & $78.5 \pm 7.9$ & 0.012 \\
\hline Sex, female & $29(42.0 \%)$ & $15(32.6 \%)$ & $14(60.9 \%)$ & 0.363 \\
\hline Affected side, left & $44(63.8 \%)$ & $30(65.2 \%)$ & $14(60.9 \%)$ & 0.793 \\
\hline \multicolumn{5}{|l|}{ Vascular risk factors } \\
\hline Hypertension & $36(52.2 \%)$ & $21(45.7 \%)$ & $15(65.2 \%)$ & 0.125 \\
\hline Atrial fibrillation & $35(50.7 \%)$ & $20(43.5 \%)$ & $15(65.2 \%)$ & 0.082 \\
\hline Diabetes & $15(21.7 \%)$ & $10(21.8 \%)$ & $5(21.8 \%)$ & 1.000 \\
\hline Dyslipidemia & $10(14.5 \%)$ & $8(17.4 \%)$ & $2(8.7 \%)$ & 0.333 \\
\hline Smoking & $21(30.4 \%)$ & $15(32.6 \%)$ & $6(26.1 \%)$ & 0.782 \\
\hline \multicolumn{5}{|l|}{ Clinical presentation } \\
\hline Preoperative NIHSS score & $20.3 \pm 5.7$ & $19.0 \pm 5.8$ & $23.0 \pm 4.8$ & 0.021 \\
\hline DWI-ASPECTS & $7.5 \pm 2.0$ & $7.9 \pm 1.7$ & $6.8 \pm 2.3$ & 0.024 \\
\hline \multicolumn{5}{|l|}{ Occlusion vessel } \\
\hline ICA & $24(34.8 \%)$ & $15(32.6 \%)$ & $9(39.1 \%)$ & 0.603 \\
\hline MCA M1 portion & 41 (59.4\%) & $28(60.9 \%)$ & $13(56.5 \%)$ & \\
\hline MCA M2 portion & $4(5.8 \%)$ & $3(6.5 \%)$ & $1(4.4 \%)$ & \\
\hline
\end{tabular}

Abbreviations: DWI-ASPETS-DWI, diffusion-weighted imaging Alberta Stroke Program Early Computed Tomography Score; ICA, internal cerebral artery; MCA, middle cerebral artery; NIHSS, National Institutes of Health Stroke Scale; SD, standard deviation.

received treatment only via the stent retriever technique, and $18(26.1 \%)$ were treated with a combination of the ADAPT and stent retriever techniques. In 30 patients (43.5\%), a TICI 3 classification was achieved. Posttreatment CT images revealed ICHs in 12 patients (14.5\%), whereas 2 (2.9\%) suffered from symptomatic ICHs.

Regarding the time courses for the good and poor outcome groups, the mean time from onset to admission was 111 and 161 minutes, respectively; the mean time from puncture to revascularization was 60 and 55 minutes, respectively; and the mean time from onset to revascularization was 244 and 302 minutes, respectively. Significant differences in the time from onset to arrival and in the time to reperfusion were noted between the outcome groups, with the times being signifi-

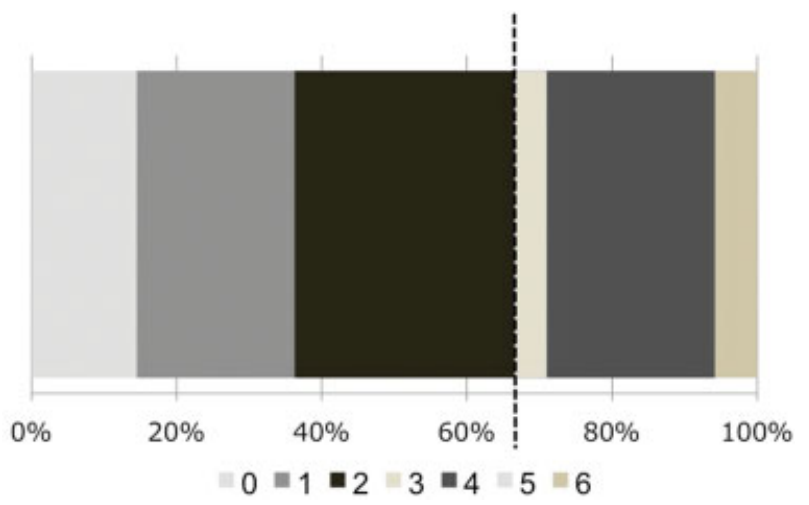

Fig. 1 Distribution of patients with each modified Rankin Scale (mRS) score at 3 months after treatment. The dashed line indicates a border between good and poor outcomes ( $\mathrm{mRS}$ of $£ 2$ ). cantly longer in the poor outcome group than in the good outcome group.

\section{Prognostic Factors}

As shown in - Tables $\mathbf{1}$ and $\mathbf{2}$, in the univariate analyses, the following variables were identified as predictors of a poor outcome at 3 months after treatment: older age, low DWIASPECTS, high preoperative NIHSS score, a longer time to admission, a longer time to reperfusion, and incomplete revascularization (TICI 2b). In the multivariate analyses, incomplete revascularization (odds ratio [OR], 4.521; 95\% confidence interval $[\mathrm{CI}], 1.143-17.883 ; p=0.026)$, preoperative NIHSS score (OR, 1.152; 95\% CI, 1.004-1.325; $p=0.028$ ), and DWIASPECTS (OR, 0.604; 95\% CI, 0.412-0.880; $p=0.003$ ) remained significant independent predictors of poor outcome at 3 months after treatment (-Table $\mathbf{3}$ ).

\section{Discussion}

The present study identified several predictors of poor outcome after successful mechanical thrombectomy, including a high NIHSS score on admission, low DWI-ASPECTS, and incomplete revascularization. These findings are consistent with those of several previous studies that evaluated the independent predictors of clinical outcome after mechanical thrombectomy in patients with acute ischemic stroke owing to intracranial large vessel occlusion, which found that a younger age, lower NIHSS score on admission, successful revascularization, and shorter procedure time were independent predictors of good outcom. ${ }^{7-10}$ 
Predictors of Poor Outcome after Successful Mechanical Thrombectomy in Patients with Acute Anterior Circulation

Table 2 Angiographic and clinical outcomes of the patients who underwent mechanical thrombectomy for acute ischemic stroke in our hospital

\begin{tabular}{|c|c|c|c|c|}
\hline & $\begin{array}{l}\text { All } \\
(n=69)\end{array}$ & $\begin{array}{l}\text { Good outcome } \\
(n=46)\end{array}$ & $\begin{array}{l}\text { Poor outcome } \\
(n=23)\end{array}$ & $p$ Value \\
\hline IV-tPA & $23(33.3 \%)$ & $18(39.1 \%)$ & $5(21.8 \%)$ & 0.183 \\
\hline \multicolumn{5}{|l|}{ Time course } \\
\hline $\begin{array}{l}\text { Time from onset to admission, } \\
\text { minutes, mean } \pm \text { SD }\end{array}$ & $128 \pm 103$ & $111 \pm 91$ & $161 \pm 120$ & 0.028 \\
\hline $\begin{array}{l}\text { Time from puncture to revascularization } \\
\text { minutes, mean } \pm S D\end{array}$ & $59 \pm 38$ & $60 \pm 41$ & $55 \pm 33$ & 0.711 \\
\hline $\begin{array}{l}\text { Time from onset to revascularization } \\
\text { minutes, mean } \pm S D\end{array}$ & $271 \pm 114$ & $244 \pm 81$ & $302 \pm 131$ & 0.028 \\
\hline \multicolumn{5}{|l|}{ Device } \\
\hline Penumbra reperfusion system & $50(72.5 \%)$ & $34(73.9 \%)$ & $16(69.6 \%)$ & 0.850 \\
\hline Stent retriever & $25(36.2 \%)$ & $15(32.6 \%)$ & $10(43.5 \%)$ & 0.742 \\
\hline Multiple devices & $18(26.1 \%)$ & $11(23.9 \%)$ & $7(30.4 \%)$ & 0.531 \\
\hline \multicolumn{5}{|l|}{ Angiographic outcome } \\
\hline $\mathrm{TICl} 2 \mathrm{~b}$ & $30(43.5 \%)$ & $15(32.6 \%)$ & $15(65.2 \%)$ & \multirow[t]{2}{*}{0.020} \\
\hline $\mathrm{TICl} 3$ & $39(56.5 \%)$ & $31(67.4 \%)$ & $8(34.8 \%)$ & \\
\hline \multicolumn{5}{|l|}{ Complication } \\
\hline Asymptomatic ICH & $10(14.5 \%)$ & $5(10.9 \%)$ & $5(21.7 \%)$ & 0.386 \\
\hline Symptomatic ICH & $2(2.9 \%)$ & $0(0 \%)$ & $2(8.7 \%)$ & 0.108 \\
\hline
\end{tabular}

Abbreviations: ICH, intracerebral hemorrhage; IV-tPA, intravenous tissue-type plasminogen activator; SD, standard deviation; TICl, Thrombolysis in Cerebral Infarction.

Here, incomplete revascularization was significantly associated with a poor clinical outcome. Several previous studies examined or mentioned potential differences in outcome between patients with a TICI of 3 versus a TICI of $2 \mathrm{~b}$. For instance, Kleine et al reported that compared with almost complete reperfusion (TICI 2 b), complete reperfusion (TICI 3 ) was associated with significantly greater neurologic recovery and better neurologic outcomes (37\% versus 68\%). ${ }^{11}$ Although not significant, Yoo et al likewise observed a higher rate of good functional outcomes ( $\mathrm{mRS}$ of $0-2$ at $~ 90$ days) in patients with

Table 3 Multivariate analysis for predictors of poor outcome

\begin{tabular}{|l|l|l|l|}
\hline & OR & $95 \% \mathrm{Cl}$ & $p$ Value \\
\hline $\begin{array}{l}\text { Preoperative } \\
\text { NIHSS score }\end{array}$ & 1.152 & $1.004-1.325$ & 0.028 \\
\hline DWI-ASPECTS & 0.604 & $0.412-0.880$ & 0.003 \\
\hline TICI 2b & 4.521 & $1.143-17.883$ & 0.026 \\
\hline Age, years & 1.053 & $0.973-1.137$ & 0.182 \\
\hline $\begin{array}{l}\text { Time from onset } \\
\text { to arrival, minutes }\end{array}$ & 1.005 & $0.991-1.024$ & 0.419 \\
\hline $\begin{array}{l}\text { Time from onset to } \\
\text { revascularization, } \\
\text { minutes }\end{array}$ & 1.002 & $0.989-1.012$ & 0.689 \\
\hline
\end{tabular}

Abbreviations: $\mathrm{Cl}$, confidence interval; DWI-ASPETS, diffusion-weighted imaging Alberta Stroke Program Early Computed Tomography Score; NIHSS, National Institute of Health Stroke Scale; OR, odds ratio; TICl, Thrombolysis in Cerebral Infarction. a TICI 3 grade compared with patients with a TICI $2 \mathrm{~b}$ grade (62\% vs. 46\%). ${ }^{12}$ The present results are in agreement with the results of these prior studies.

In our study, a grade of $\mathrm{TICI} 2 \mathrm{~b}$ indicated a reperfused territory of $>50 \% .^{13}$ In other words, the reperfused territory in TICI 2b patients varies from 50 to $99 \%$, resulting in different final infarct volumes. Previously, Zaidi et al reported that the final infarct volume is a predictor of outcome. ${ }^{14}$ In their study, patients with a TICI grade of 3 had smaller infarct volumes than did patients with a TICI grade of $2 \mathrm{~b} .{ }^{14}$ Our study also found that a low DWI-ASPECTS was predictive of a poor outcome. The tissue changes described by the score most likely represent irreversible brain damage. Although our study did not calculate the infarct volume, the DWI-ASPECTS is likely closely related to the infarct volume. Therefore, clinicians should attempt to achieve complete revascularization to reduce the infarct volume, especially in patients with high DWI-ASPECTS, as this may help increase the number of patients with a good outcome.

The NIHSS score is a quick and relatively simple method of estimating the extent and severity of a stroke. ${ }^{15}$ Higher NIHSS scores are associated with the presence of more proximal vessel occlusions and poor collaterals. ${ }^{16}$ In our study, the NIHSS score at admission was an independent predictor of a favorable outcome. This finding is consistent with other studies examining patient outcomes after mechanical thrombectomy. For example, Costalat et al reported that the baseline NIHSS score was an independent predictor of poor outcome at 3 months after treatment. ${ }^{9}$ Similarly, Jiang et al showed that fewer patients with baseline NIHSS scores $<20$ had a good outcome 


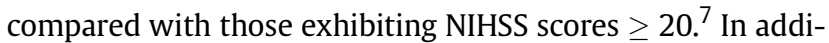
tion, Shi et al reported that the baseline NIHSS score was an independent predictor of functional dependence (mRS of 3-6) despite successful revascularization in 109 patients treated with the Trevo stent-retriever system. ${ }^{17}$ However, the high NIHSS score should not be used as an exclusion criterion for acute anterior circulation stroke, as patients with severe stroke often have worse outcomes with medical therapy alone.

Finally, our study demonstrated that the time from onset to revascularization was longer in the poor outcome group than it was in the good outcome group. This result was consistent with the findings of a previous study, which revealed an association between the time from onset to revascularization and a favorable clinical outcome and survival. ${ }^{18}$ Given that no significant differences in the time from puncture to revascularization was identified between the groups, minimizing delays between stroke onset and hospital admission is a key factor for achieving good clinical outcomes.

The present study has some limitations. First, the sample size was small and the evaluations were only performed at a single institution. Second, as mentioned previously, this was a retrospective and noncontrolled study with a bias in patient selection. Last, we only used the modified TICI score. Another grade, namely TICI 2c, exists and is defined as "nearcomplete perfusion except for slow flow in a few distal cortical vessels, or presence of small distal cortical emboli."19 Furthermore, Almekhlafi et al reported that a combined criterion subsuming TICI 2c/3 as "successful" revascularization might be a more accurate predictor of a favorable clinical outcome than the widely applied TICI $2 \mathrm{~b} / 3$ criterion. $^{20}$ Further prospective studies using the TICI 2c category will be required to determine which grade best predicts patients' outcomes.

\section{Conclusion}

Our findings show that the preoperative NIHSS score, DWIASPECTS, and incomplete revascularization (TICI $2 \mathrm{~b}$ ) are predictors of poor outcome despite successful mechanical thrombectomy for acute anterior circulation stroke. These results imply that clinicians should try to achieve complete revascularization to reduce the infarct volume, especially in patients with a high DWI-ASPECTS, as this has the potential to increase the number of patients with a good outcome.

\section{Conflict of Interest}

None.

\section{References}

1 Berkhemer OA, Fransen PS, Beumer D, et al; MR CLEAN Investigators. A randomized trial of intraarterial treatment for acute ischemic stroke. N Engl J Med 2015;372(01):11-20

2 Goyal M, Demchuk AM, Menon BK, et al; ESCAPE Trial Investigators. Randomized assessment of rapid endovascular treatment of ischemic stroke. N Engl J Med 2015;372(11):1019-1030
3 Saver JL, Goyal M, Bonafe A, et al; SWIFT PRIME Investigators. Stentretriever thrombectomy after intravenous t-PA vs. t-PA alone in stroke. N Engl J Med 2015;372(24):2285-2295

4 Campbell BC, Mitchell PJ, Kleinig TJ, et al; EXTEND-IA Investigators. Endovascular therapy for ischemic stroke with perfusionimaging selection. N Engl J Med 2015;372(11):1009-1018

5 Jovin TG, Chamorro A, Cobo E, et al; REVASCAT Trial Investigators. Thrombectomy within 8 hours after symptom onset in ischemic stroke. N Engl J Med 2015;372(24):2296-2306

6 Goyal M, Menon BK, van Zwam WH, et al; HERMES collaborators. Endovascular thrombectomy after large-vessel ischaemic stroke: a meta-analysis of individual patient data from five randomised trials. Lancet 2016;387(10029):1723-1731

7 Jiang S, Fei A, Peng Y, et al. Predictors of outcome and hemorrhage in patients undergoing endovascular therapy with solitaire stent for acute ischemic stroke. PLoS One 2015;10(12):e0144452

8 Yoon W, Kim SK, Park MS, Baek BH, Lee YY. Predictive factors for good outcome and mortality after stent-retriever thrombectomy in patients with acute anterior circulation stroke. J Stroke 2017;19(01):97-103

9 Costalat V, Lobotesis K, Machi P, et al. Prognostic factors related to clinical outcome following thrombectomy in ischemic stroke (RECOST study). 50 patients prospective study. Eur J Radiol 2012; 81(12):4075-4082

10 Daou B, Chalouhi N, Starke RM, et al. Predictors of outcome, complications, and recanalization of the solitaire device: a study of 89 cases. Neurosurgery 2015;77(03):355-360, discussion 360-361

11 Kleine JF, Wunderlich S, Zimmer C, Kaesmacher J. Time to redefine success? TICI 3 versus TICI $2 \mathrm{~b}$ recanalization in middle cerebral artery occlusion treated with thrombectomy. J Neurointerv Surg 2017;9(02):117-121

12 Yoo AJ, Simonsen CZ, Prabhakaran S, et al; Cerebral Angiographic Revascularization Grading Collaborators. Refining angiographic biomarkers of revascularization: improving outcome prediction after intra-arterial therapy. Stroke 2013;44(09):2509-2512

13 Tomsick T, Broderick J, Carrozella J, et al; Interventional Management of Stroke II Investigators. Revascularization results in the Interventional Management of Stroke II trial. AJNR Am J Neuroradiol 2008;29(03):582-587

14 Zaidi SF, Aghaebrahim A, Urra X, et al. Final infarct volume is a stronger predictor of outcome than recanalization in patients with proximal middle cerebral artery occlusion treated with endovascular therapy. Stroke 2012;43(12):3238-3244

15 Jauch EC, Saver JL, Adams HP Jr, et al; American Heart Association Stroke Council; Council on Cardiovascular Nursing; Council on Peripheral Vascular Disease; Council on Clinical Cardiology. Guidelines for the early management of patients with acute ischemic stroke: a guideline for healthcare professionals from the American Heart Association/American Stroke Association. Stroke 2013;44(03):870-947

16 Leigh R, Zaidat OO, Suri MF, et al. Predictors of hyperacute clinical worsening in ischemic stroke patients receiving thrombolytic therapy. Stroke 2004;35(08):1903-1907

17 Shi ZS, Liebeskind DS, Xiang B, et al; Multi MERCI, TREVO, and TREVO 2 Investigators. Predictors of functional dependence despite successful revascularization in large-vessel occlusion strokes. Stroke 2014; 45(07):1977-1984

18 Mazighi M, Chaudhry SA, Ribo M, et al. Impact of onset-to-reperfusion time on stroke mortality: a collaborative pooled analysis. Circulation 2013;127(19):1980-1985

19 Goyal M, Fargen KM, Turk AS, et al. 2C or not 2C: defining an improved revascularization grading scale and the need for standardization of angiography outcomes in stroke trials. J Neurointerv Surg 2014;6(02):83-86

20 Almekhlafi MA, Mishra S, Desai JA, et al. Not all "successful" angiographic reperfusion patients are an equal validation of a modified TICI scoring system. Interv Neuroradiol 2014;20(01):21-27 